\title{
Manipulation of antioxidant and glycaemic properties of extruded rice based breakfast cereal products using pomelo fruit by-product material
}

\author{
N. Shi ${ }^{1}$, J.O. Narciso ${ }^{1}$, X. Gou ${ }^{2}$, M.A. Brennan ${ }^{1}$, X.-A. Zeng ${ }^{2}$ and C.S. Brennan ${ }^{1 *}$ \\ ${ }^{1}$ Centre for Food Research and Innovation, Department of Wine, Food and Molecular Biosciences, Lincoln University, 7647 \\ Lincoln, New Zealand; ${ }^{2}$ College of Light Industry and Food Science, South China University of Technology, Guangzhou \\ 510640, Guangdong, China P.R.; charles.brennan@lincoln.ac.nz
}

Received: 24 January 2017 / Accepted: 29 May 2017

(c) 2017 Wageningen Academic Publishers

OPEN ACCESS CC) (i) (2) RESEARCH ARTICLE

\begin{abstract}
Pomelo (Citrus grandis) is a citrus fruit native to Southeast Asia, and is considered a good source of dietary fibre and antioxidant materials. The outside of the pomelo fruit (the peel and the pith) contains high levels of pectin, which has been shown to lower blood sugar and cholesterol. This work evaluated the ability to lower the glycaemic impact of rice-based breakfast cereal by the inclusion of pomelo rind material. Pomelo rind material was included at rates of $0,5,10$, and $15 \%$ in rice-based cereal. Total phenols, glycaemic response, moisture, starch, texture, expansion and colour of the pomelo enriched extrudates were measured. As the inclusion of pomelo rind increased, the moisture content (dry matter based) decreased. The expansion ratio decreased as inclusion of pomelo rind increased: for instance 5,10 , and $15 \%$ showed significantly lower expansion ratios $(P<0.05)$ than the brown rice control sample. Colour also became darker with increased inclusion of pomelo rind. The results from in vitro analysis illustrate a reduction in the potential glycaemic response with increasing levels of pomelo rind inclusion. Antioxidant assays using total phenolic content and oxygen radical absorbance capacity revealed that pomelo rind inclusion increased the antioxidant activity of the extrudates. The study illustrates the potential of pomelo by-product stream utilisation in manipulating the nutritional value and health benefits of rice based extruded breakfast cereal products.
\end{abstract}

Keywords: pomelo, extrusion, glycaemic index, anti-oxidant, breakfast cereal

\section{Introduction}

Extrusion is one of the most important processes for producing breakfast cereals and snack bars (Brennan et al., 2011). Breakfast cereal has been shown to have a high glycaemic impact (Brand-Miller et al., 2002). In addition, researchers have indicated that extruded snack products are generally energy dense (high glycaemic response type foods) and may be low in bioactive nutrients (Robin et al., $2015,2016)$. Thus there has been a plethora of research in recent years investigating how new food ingredients could be included into extruded snack products to improve their nutritional quality including legumes, vegetables and fruit (Oliveiera et al., 2015; Osen et al., 2015; Rashid et al., 2015; Singh et al., 2016). Citrus fruits are generally rich in dietary fibre as well as phenolic compounds (Barker, 1994). The world production of citrus fruits is nearly 80 million tonnes per year (Bocco et al., 1998). In the last decade, demand for quality juice beverages has increased within the food industry. Due to the enormous waste in fruit juice industry and also the demand for natural ingredients in food, using fruit by-products in other manufactured foods is becoming a new trend (Lohani and Muthukumarappan, 2016; Rohm et al., 2015). Research has indicated that fruit waste accounts for approximately $15 \%$ of grapes and up to $50 \%$ of citrus fruits (Rohm et al., 2015). This is a large amount of fruit by-products formed each year.

Pomelo (Citrus grandis) in the family of Rutaceae, is a citrus fruit resembling a big grapefruit. Pomelo is native to South and Southeast Asia. It is usually pale green to yellow, with very thick rind pith with sweet flesh and contains carotenoids, phenolics, vitamin C, lycopene, and anthocyanins. The phenolic acid and other compounds 
contained in the fruit allow it to act as a natural antioxidant. Pomelo rind and pith are rich in pectin, which helps lower blood sugar and cholesterol, with approximately 50\% fibre yield extracted from the fruit peel (Min et al., 2003). Previous research has investigated the use of pomelo pith in noodle production and quality (Wandee et al., 2014).

The cereal food industry has been interested in adding fibre to cereal foods for a number of years but this fortification can result in reduction in quality such as lack of consumer acceptance of texture and appearance (Foschia et al., 2015; Grigor et al., 2016). This is despite the fact that fibre-rich cereal products can exert significant reductions in starch digestibility and glucose release values using both in vitro and in vivo digestion procedures (Brennan et al., 2012). Some of this problem may arise from the interactions between protein, starch and lipids in food systems (De Pilli et al., 2015, 2016; Gulati et al., 2016). It was the aim of this research to improve the health potential of rice-based breakfast cereals using the pomelo rind pith, which is the by-product of pomelo fruit.

\section{Methods and materials}

Long grain brown rice from Thailand (Budget, Safeway Trades Ltd, Auckland, NZ) was used in the experiment. Fresh pomelo used in the project was bought from a vegetable supermarket in New Zealand, which was imported from China. The outer layers of the pomelo rind were removed from the central fruit. This peel included the outer waxy layer and the pithy sub layer. Peel was ovendried at $50{ }^{\circ} \mathrm{C}$ overnight, and 5,10 , and $15 \%$ inclusion in brown rice were prepared (Table 1 ).

\section{Manufacture of extruded breakfast cereals}

Extrusion was conducted in a single screw extruder (screw length: $30 \mathrm{~cm}$, diameter: $30 \mathrm{~mm}$ ) through a $3 \mathrm{~mm}$ die face and collected as collets (Northern finance Ltd, Auckland, New Zealand). The temperature at the die face was $180{ }^{\circ} \mathrm{C}$ with the temperature not controlled, the torque of the process is summarised in Table 2 and a screw speed of 200 rpm was maintained during the process with a feed rate of $9 \mathrm{~kg} / \mathrm{h}$. Extruded products were collected, cooled and

Table 1. Recipes used to create extruded breakfast cereal products.

$\begin{array}{lll}\text { Substance level } & \text { Brown rice }(g) & \text { Dried pomelo rind }(\mathbf{g}) \\ \text { Control } & 500 & 0 \\ 5 \% & 475 & 25 \\ 10 \% & 450 & 50 \\ 15 \% & 425 & 75\end{array}$

then stored in sealed plastic bags at room temperature for further analysis.

\section{Physicochemical properties of cereal products}

\section{Moisture}

Moisture determination of raw pomelo rind and extruded products was conducted according to the AACC methodology (moisture-air oven methods, method 4415. 02) (AACC, 2010) and moisture loss during extrusion was calculated.

\section{Expansion}

The diameter of 20 extruded collets was measured using an electronic Caliper Series 1112 (Insize Ltd, Loganville, GA, USA). Percentage expansion of the samples was determined using the formula:

$\%$ expansion $=($ average cereal extrudate $\times 100) /$ die diameter

\section{Colour}

The sample colour was measured using a Minolta CR 300 colourimeter (Konica Minolta Sensing Inc., Osaka, Japan) and expressed as Hunter $L * a^{*} b *$ values ( $L *$ defines the lightness, $a^{*}$ value the red-greenness and $b^{*}$ value the blueyellowness).

\section{Texture analysis}

Product hardness and crispiness were determined using a Stable Microsystems Texture Analyzer (TA-XT32, Stable Micro Systems, Surrey, UK). An aluminium cylinder probe of $35 \mathrm{~mm}$ diameter was used with a test speed of $1 \mathrm{~mm} / \mathrm{s}$ with a trigger force of $5 \mathrm{~g}$. Extruded pellets were axially compressed to $50 \%$ of their original height, and the maximum force recorded during the compression was expressed as the hardness of the product. Hardness of the product was defined as the peak force exhibited on compression of the sample, whereas crispiness was defined as the number of fracture peaks $>50 \mathrm{~g}$ obtained during analysis. All measurements were performed on 20 collets.

Table. 2. Processing parameters of the extruded cereals.

$\begin{array}{lll}\text { Sample } & \text { Current amps } & \text { Torque }(\mathrm{g} / \mathrm{m}) \\ \text { Control } & 5.25 & 50.0 \\ 5 \% & 4.87 & 39.1 \\ 10 \% & 5.07 & 38.2 \\ 15 \% & 4.89 & 35.7\end{array}$


Starch

Total starch of the extrudates was determined in triplicates using the Total Starch assay kit from Megazyme International (Wicklow, Ireland), following the manufacturer's protocol.

\section{In vitro starch hydrolysis}

Extruded products were finely milled. The amount of glucose released over $120 \mathrm{~min}$ by in vitro digestion was determined (Gao et al., 2016). This method replicates human digestion and reducing sugars are measured using the dinitrosalicylic acid method measuring absorbance at $530 \mathrm{~nm}$.

\section{Anti-oxidant properties}

Sample extractions were prepared for total phenolic content (TPC), and oxygen radical absorbance capacity (ORAC) with minor modifications on the percentage methanol used. One gram of ground sample was mixed with $20 \mathrm{ml}$ of $70 \%$ methanol ( $50 \%$ methanol for ORAC), overnight on a magnetic agitator.

\section{Total phenolic content}

TPC of the samples were measured using $0.2 \mathrm{~N}$ FolinCiocalteu (FC) reagent (Sigma, St Louis, MO, USA) according to the method adapted from Singleton and Rossi (1965). The absorbance was read at $760 \mathrm{~nm}$ (V1200 spectrophotometer, Global Science, Auckland, New Zealand). Gallic acid (25-200 $\mu \mathrm{g})$ (Sigma-Aldrich, Steinheim, Germany) was used for the standard curve and TPC was expressed as gallic acid equivalent per gram sample (GAE/g sample).

\section{Oxygen radical absorbance capacity assay}

The ORAC-fluorescence assay was performed according to the method described in (Thaipong et al., 2006). The measurement was carried out using FLUOstar OPTIMA plate reader (BMG Labtech GmbH, Offenburg, Germany). Data was analysed using MARS software (V1.20, BMG Labtech $\mathrm{GmbH}$ ) and the area under curve (AUC) was obtained. The ORAC assay results were expressed as $\mu \mathrm{mol}$ $\mathrm{TE} / \mathrm{g}$ sample.

\section{Statistical analyses}

All analysis was conducted in triplicate unless otherwise stated. Statistical analyses were performed using Minitab (Minitab, Inc., State College, PA, USA) to calculate the means and standard deviation. Tukey's methods were used to determine the $P$-value. Significant differences were reported as $(P \leq 0.05)$.

\section{Results and discussion}

\section{Moisture}

Moisture loss is defined as the final product compared to that in the original mixture before extrusion. As the inclusion of pomelo rind increased the moisture content decreased. A higher amount of moisture was lost from the brown rice control compared to the inclusion of dried pomelo rind possibly due to the higher levels of dietary fibre present in the pomelo rind absorbing and retaining greater levels of moisture compared to rice grains. The inclusion of 5,10 , and $15 \%$ pomelo rind had no significant difference $(P>0.05)$ in moisture loss (Table 3$)$.

\section{Expansion ratio}

The expansion characteristics of extruded products had a significant impact on the acceptability of the final product. The expansion ratio is a product of the degree of puffing undergone by the mixture of brown rice and pomelo rind as it exits the extruder (Thymi et al., 2005; Yağcl and Göğüş, 2008).

Expansion ratio of extruded cereals with different inclusion rates of pomelo rind is shown in Table 3. Extruded cereals with pomelo rind inclusion at 5, 10, and 15\% level exhibited a significantly lower expansion ratio compared to control extruded brown rice $(P<0.05)$. Similar findings were observed on values of both wheat and rice-based extrudates using inclusions of dietary fibres with the researchers indicating a relationship between the amount of fibre in an extruded food product, greater water retention and a subsequent reduction in expansion ratio with an increase in product density (Asare et al., 2004; Brennan et al., 2008). Extruded cereals with $10 \%$ pomelo rind inclusion rate also exhibited a lower expansion ratio than the control samples and the 5\% inclusion sample; however, this was not statistically different. Extruded cereals with a 10\% pomelo rind inclusion rate exhibited a lower expansion ratio than the control samples and 5\% sample; however, this was higher than the $15 \%$ sample due to unevenly distributed pomelo rind during the extrusion process.

Table 3. Moisture content $(n=3)$ and expansion ratio $(n=20)$ for pomelo rind inclusion extrudates. ${ }^{1}$

$\begin{array}{lll} & \text { Moisture content (\%) } & \text { Expansion ratio (\%) } \\ \text { Control } & 7.8^{\mathrm{a}} & 333.1 \pm 0.5^{\mathrm{a}} \\ 5 \% & 7.4^{\mathrm{b}} & 291.5 \pm 0.6^{\mathrm{b}} \\ 10 \% & 7.4^{\mathrm{b}} & 281.2 \pm 0.8^{\mathrm{c}} \\ 15 \% & 6.7^{\mathrm{c}} & 285.3 \pm 0.8^{\mathrm{c}}\end{array}$




\section{Colour}

An important appearance feature of cereal breakfast to consumers is the surface colour of the extruded products, which is related to the percentage inclusion of pomelo rind during the extrusion with brown rice. The picture of ground samples is shown in appendix. The control samples with no pomelo rind added was the lightest. As the inclusion of pomelo rind increased in the sample, the colour became darker, suggesting that the pomelo rind had greater impact on the colour of breakfast cereal. The lightness $\left(\mathrm{L}^{*}\right)$ of control sample was $97.68 \pm 0.18$ the highest value, which indicates the presence of $\beta$-carotene (17). The lightness of all the four samples was similar to each other; however, the redness ( $\left.\mathrm{a}^{*}\right)$ decreased (from positive to negative) as the inclusion of pomelo rind increased. The yellowness $\left(b^{*}\right)$ increased (from negative to positive) as the inclusion of pomelo rind increased (Figure 1).

\section{Textural analysis}

The textural properties of extruded products are generally described by the hardness and crunchiness (Brennan et al., 2008). The hardness of the extrudates is associated with the expansion and cell structure of the product, and is represented by the maximum peak force. The hardness pertains to the resistance of the extruded products to the initial penetration.

The hardness of extruded pomelo rind enriched cereals were similar to the control samples. The crispiness of the extruded breakfast cereal increased as the inclusion of pomelo rind increased (Table 4). The hardness and crispiness are both higher than those reported by previous researchers (Brennan et al., 2012). This could be due to the hardness of dried pomelo rind, which contains more fibre in the peel.

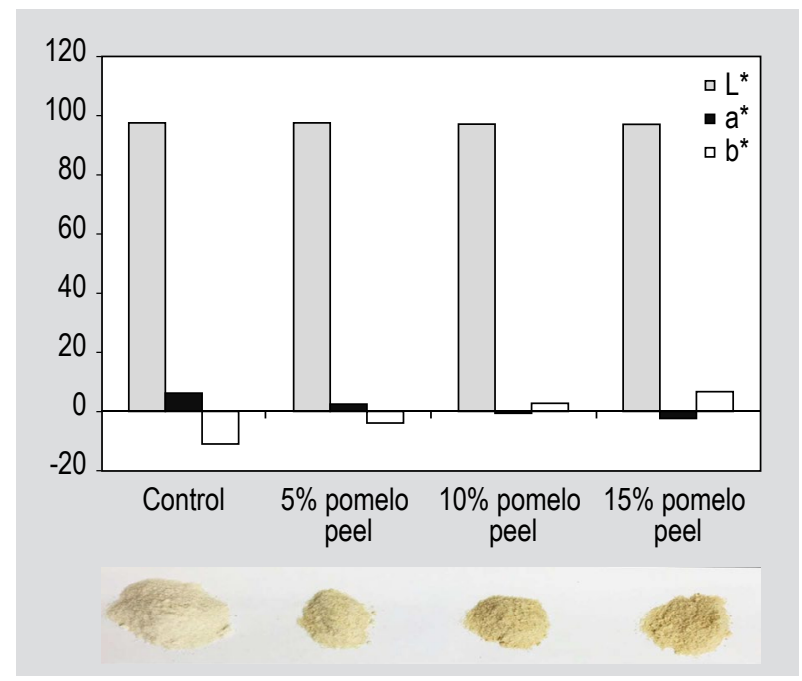

Figure 1. Colour measurement (Hunter $L^{*} a^{*} b^{*}$ values) of extruded cereals $(n=3)$.
Table 4. Textural properties of extruded cereal containing pomelo rind.

\begin{tabular}{lll} 
& Hardness $(\mathrm{g})$ & \multicolumn{1}{c}{ Crispiness (no. of peaks) $^{1}$} \\
Control & $39.7 \pm 4.1$ & $3,463 \pm 1,183^{\mathrm{a}}$ \\
$5 \%$ & $41.1 \pm 4.0$ & $2,641 \pm 530^{\mathrm{b}}$ \\
$10 \%$ & $37.4 \pm 5.7$ & $3,434 \pm 847^{\mathrm{a}}$ \\
$15 \%$ & $38.6 \pm 5.8$ & $4,397 \pm 719$ \\
\hline${ }^{1}$ Columns with different letters represent significant differences $(P<0.05)$.
\end{tabular}

\section{Starch content}

The starch content of plain brown rice was $69.3 \%$, at $5 \%$ inclusion of pomelo rind was $68.4 \%$, at $10 \%$ inclusion was $63.09 \%$ and at $15 \%$ was $61.29 \%$. The starch content decreased as the inclusion of pomelo rind increased. However, the starch content of $5 \%$ inclusion of pomelo rind showed no significant difference $(P>0.05)$ to the control sample $(\mathrm{R}=0.5799)$.

Research illustrates the fact that fibre-rich products can exert significant reductions in starch digestibility and glucose release values using both in vitro and in vivo digestion procedures (Brennan et al., 2008, 2012; Symons and Brennan, 2004). Previously researchers have reported that $51 \%$ fibre yield extracted from the grapefruit peel (Min et al., 2003). Peels are fibre-rich products, and therefore, addition of pomelo rind to the extruded products showed decreased starch content.

\section{In vitro starch digestion}

Higher inclusion rates of pomelo rind led to greater reductions in glucose released at all time points except for at the $15 \%$ inclusion rate of pomelo rind which produced less reducing sugar during the in vitro digestion process. Previous research has indicated that whilst the inclusion of fibre rich products into cereal foods may lead to a reduction in predictive glycaemic impact and starch degradation, it is too simplistic to assert that all dietary fibres behave in a similar way (Brennan et al., 2005). For instance dietary fibres from different plant species comprise of different soluble and insoluble fractions; it is the ratios of these fibre fractions in a food structure that manipulate the starch degradation process by altering the rate of enzyme reactions during the digestion process. The average AUC values for $5 \%$ (572.8 \pm 18.4$)$, and $10 \%(579.8 \pm 11.7)$ pomelo rind inclusion showed no significant $(P>0.05)$ difference with the control sample (570.8 \pm 19.3$)$. Only the $15 \%$ pomelo rind inclusion exhibited reduced amount of reducing sugars released (545.9 \pm 20.6$)$ (Figure 2). This observation can be explained by an increase in the amount of fibre in the products. 
Pomelo rind contains pectin, which helps lower blood sugar and cholesterol. Therefore, $15 \%$ inclusion of pomelo rind had a greater impact on the glycaemic response on the extruded breakfast cereal. Previous reports investigating the effect of the inclusion of mushroom by-product material also suggested a similar finding, in that 15\% mushroom enriched cereal extruded samples was reduced by $29 \%$ whilst lower levels did not show such a significant decrease (Brennan et al., 2012).

\section{Anti-oxidant activity}

The antioxidant activity of the pomelo rind extrudates was evaluated both by TPC and ORAC. The $15 \%$ pomelo rind had the highest TPC $(148.0 \pm 1.9 \mu \mathrm{g} \mathrm{GAE} / \mathrm{g}$ wet weight), followed by $10 \%$ pomelo rind $(128.2 \pm 4.8 \mu \mathrm{g} \mathrm{GAE} / \mathrm{g}$ wet weight), $5 \%$ pomelo rind ( $77.9 \pm 4.0 \mu \mathrm{g} \mathrm{GAE} / \mathrm{g}$ wet weight), and lastly, by the brown rice control $(30.6 \pm 0.1 \mu \mathrm{g}$ GAE/g wet weight) (Figure 3 ). This trend could be attributed to the increasing amount of phenolics in the sample as more pomelo rind was included in the extrudates.

In the ORAC assay, the pomelo rind significantly increased the scavenging activity; the rice control has no detectable antioxidant activity, while $10 \%$ pomelo rind, and $15 \%$ pomelo rind exhibited similar scavenging activity (Figure 4). Inclusion of pomelo rind in extrudates appears to increase the anti-oxidant capacity of the food sample compared to the brown rice control in an ORAC assay; plotting the TPC against the ORAC activity obtained for the extrudates shows a positive linear correlation $\left(\mathrm{R}^{2}=0.98\right)$. It suggests that increasing TPC leads to higher ORAC activity. ORAC assay is considered a relevant assessment of anti-oxidant level in a biological system (Roy et al., 2010).

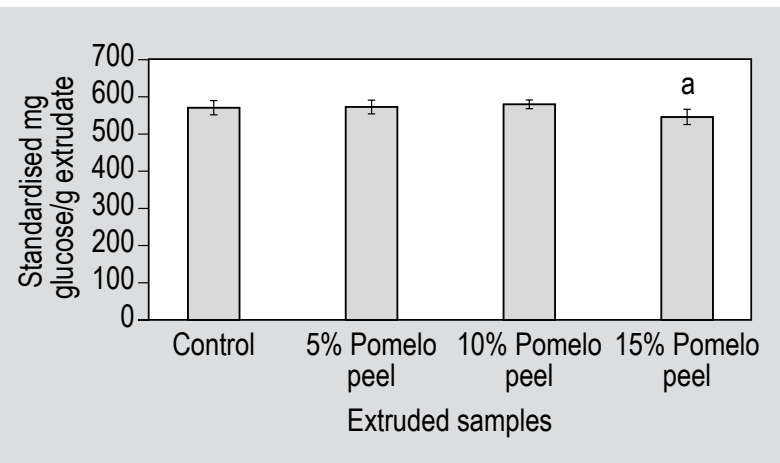

Figure 2. The amount of glucose released during the extrusion process of different inclusion of pomelo rind. Columns with different letters represent significant differences $(P<0.05)$.

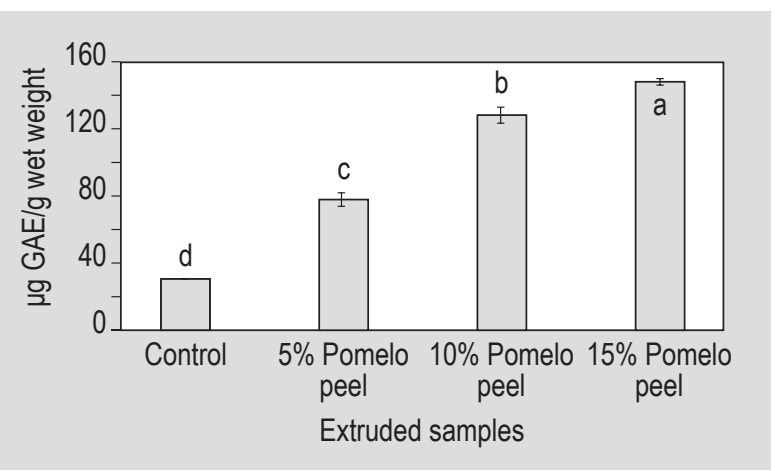

Figure 3. TPC of brown rice control, $5 \%$ pomelo rind, $10 \%$ pomelo rind, and $15 \%$ pomelo rind inclusion in extrudates using the Folin-Ciocalteau reagent $(n=9)$. Columns with different letters represent significant differences $(P<0.05) . \mathrm{GAE}=$ gallic acid equivalent.

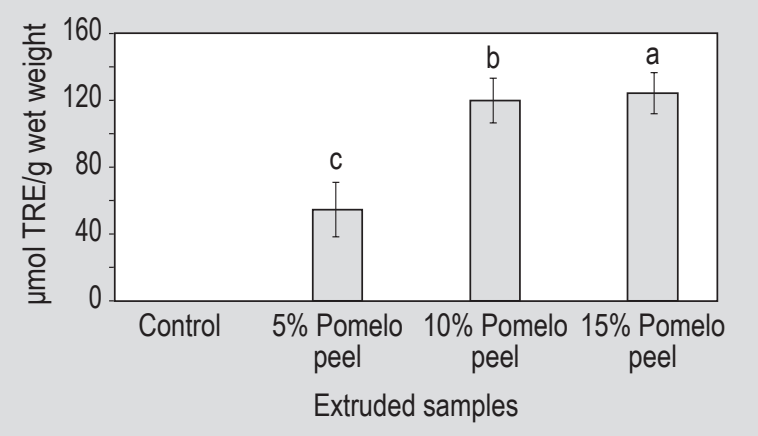

Figure 4. Antioxidant activity of brown rice control and pomelo rind extrudates in the oxygen radical absorbance capacity assay, expressed as $\mu \mathrm{mol}$ TRE/g wet weight. $(n=9)$. Columns with different letters represent significant differences $(P<0.05)$. $\mathrm{TRE}=$ total response extractable.

\section{Conclusions}

This study is the first research to evaluate the potential use of pomelo waste product in developing a health enhanced breakfast cereal product. As the inclusion of pomelo rind increased, the moisture and expansion rate were decreased. The colour was darker with increased of pomelo rind in the extruded products. The starch content of each product was decreased as the increase of pomelo rind. The digestibility of starch in the cereal also decreased at the $15 \%$ inclusion rate compared to the control, illustrating that the inclusion of pomelo rind can reduce the predicted glycaemic response of extruded product. In particular, $15 \%$ pomelo rind inclusion shows a significantly lower AUC values compared to control brown rice sample. Aside from the glycaemic response, inclusion of pomelo rind from 10 or $15 \%$ results in significant increase in antioxidant property of the pomelo rind extrudates compared to the brown rice control. The research is therefore significant in realising the potential 
of improving the nutritional quality of cereal extruded products by incorporating citrus by-product components.

\section{Acknowledgements}

This research was supported by S\&T projects of Guangdong Province (2013B091100004,2015A030312001 and 2017B020207001).

\section{References}

American Association of Cereal Chemists International (AACC), 2010. Moisture-air ovens method (method 44-15.02). AACC, St. Paul, MN, USA.

Asare, E.K., Sefa-Dedeh, S., Sakyi-Dawson, E. and Afoakwa, E.O., 2004. Application of response surface methodology for studying the product characteristics of extruded rice-cowpea-groundnut blends. International Journal of Food Science and Nutrition 55: 431-439.

Baker, R.A., 1994. Potential dietary benefits of citrus pectin and fiber. US Citrus and Subtropical Products Lab., Vol. 48, USDA-ARS, 600 Ave. S, Winter Haven, FL 33880, USA, pp. 133.

Bocco, A., Cuvelier, M.-E., Richard, H. and Berset, C., 1998. Antioxidant activity and phenolic composition of citrus peel and seed extracts. Journal of Agricultural and Food Chemistry 46: 2123-2129.

Brand-Miller, J., Foster-Powell, K. and Holt, S.H.A., 2002. International table of glycemic index and glycemic load values. American Journal of Clinical Nutrition 76(1): 5-56.

Brennan, C., Brennan, M., Derbyshire, S. and Tiwari, B.K., 2011. Effects of extrusion on the polyphenols, vitamins and antioxidant activity of foods. Trends in Food Science and Technology 22(10): 570-575.

Brennan, M.A., Derbyshire, E., Tiwari, B.K. and Brennan, C.S., 2012. Enrichment of extruded snack products with copropducts from chestnut mushroom (Agrocybe aegerita) production: interactions between dietary fiber, physicochemical characteristics, and glycemic load. Journal of Agricultural and Food Chemistry 60: 4396-4401.

Brennan, M.A., Monro, J.A. and Brennan, C.S., 2008. Effect of inclusion of soluble and insoluble fibers into extruded breakfast cereal products made with reverse screw configuration. International Journal of Food Science and Technology 43: 2278-2288.

De Pilli, T., Giuliani, R., Buléon, A., Pontoire, B. and Legrand, J., 2016. Effects of protein-lipid and starch-lipid complexes on textural characteristics of extrudates based on wheat flour with the addition of oleic acid. International Journal of Food Science and Technology 51: 1063-1074.

De Pilli, T., Legrand, J., Derossi, A. and Severini, C., 2015. Effect of proteins on the formation of starch lipid complexes during extrusion cooking of wheat flour with the addition of oleic acid. International Journal of Food Science and Technology 50: 515-521.

Foschia, M., Peressini, D., Sensidoni, A., Brennan, M.A. and Brennan, C.S., 2015. Synergistic effect of different dietary fibers in pasta on in vitro starch digestion. Food Chemistry 172: 245-250.

Gao, J., Brennan, M.A., Mason, S.L. and Brennan, C.S., 2016. Effect of sugar replacement with stevianna and inulin on the texture and predictive glycaemic response of muffins. International Journal of Food Science and Technology 51: 1979-1987.
Grigor, J.M., Brennan, C.S., Hutchings, S.C. and Rowlands, D.S., 2016. The sensory acceptance of fibre-enriched cereal foods: a metaanalysis. International Journal of Food Science and Technology 51: 3-13.

Gulati, P., Weier, S.A., Santra, D., Subbiah, J. and Rose, D.J., 2016. Effects of feed moisture and extruder screw speed and temperature on physical characteristics and antioxidant activity of extruded proso millet (Panicum miliaceum) flour. International Journal of Food Science and Technology 51: 114-122.

Lohani, U.C. and Muthukumarappan, K., 2016. Effect of sequential treatments of fermentation and ultrasonication followed by extrusion on bioactive content of apple pomace and textural, functional properties of its extrudates. International Journal of Food Science and Technology 51: 1811-1819.

Min, G.L., Yingming, P., Ming-Ming, T., Heng-Jian, Y., 2003. Study on extraction technology of dietary fiber from shaddock peel. Technology and Development of Chemical Industry 32(6): 21.

Oliveira, L.C., Rosell, C.M. and Steel, C.J., 2015. Effect of the addition of whole-grain wheat flour and of extrusion process parameters on dietary fiber content, starch transformation and mechanical properties of a ready-to-eat breakfast cereal. International Journal of Food Science and Technology 50: 1504-1514.

Osen, R., Toelstede, S., Eisner, P. and Schweiggert-Weisz, U., 2015. Effect of high moisture extrusion cooking on protein-protein interactions of pea (Pisum sativum L.) protein isolates. International Journal of Food Science and Technology 50: 1390-1396.

Rashid, S., Rakha, A., Anjum, F.M., Ahmed, W. and Sohail, M., 2015. Effects of extrusion cooking on the dietary fiber content and Water Solubility Index of wheat bran extrudates. International Journal of Food Science and Technology 50: 1533-1537.

Robin, F., Heindel, C., Pineau, N., Srichuwong, S. and Lehmann, U., 2016. Effect of maize type and extrusion-cooking conditions on starch digestibility profiles. International Journal of Food Science and Technology 51: 1319-1326.

Robin, F., Théoduloz, C. and Srichuwong, S., 2015. Properties of extruded whole grain cereals and pseudocereals flours. International Journal of Food Science and Technology 50: 2152-2159.

Rohm, H., Brennan, C., Turner, C., Gunther, E., Campbell, G., Hernando, I., Struck, S. and Kontogiorgos, V., 2015. Adding value to fruit processing waste: innovative ways to incorporate fibers from berry pomace in baked and extruded cereal-based foods - A SUSFOOD project. Foods 4: 690-679.

Roy, M.K., Koide, M., Rao, T.P., Okubo, T., Ogasawara, Y. and Juneja, L.R., 2010. ORAC and DPPH assay comparison to assess antioxidant capacity of tea infusions: relationship between total polyphenol and individual catechin content. International Journal of Food Science and Nutrition 61(2): 109-124.

Singh, J.P., Kaur, A., Shevkani, K., Singh, N. and Singh, B., 2016. Physicochemical characterisation of corn extrudates prepared with varying levels of beetroot (Beta vulgaris) at different extrusion temperatures. International Journal of Food Science and Technology 51: 911-919.

Singleton, V.L. and Rossi, J.A., 1965. Colourimetry of total phenolics with phosphomolybdic phosphotungstic acid reagents. American Journal of Enology and Viticulture 16: 144-158. 
Symons, L.J. and Brennan, C.S., 2004. The effect of barley $\beta$-glucan fiber fractions on starch gelatinization and pasting characteristics. Journal of Food Science 69: 257-260.

Thaipong, K., Boonprakob, U., Crosby, K., Cisneros-Zevallos, L. and Byrne, H.D., 2006. Comparison of ABTS, DPPH, FRAP and ORAC assays for estimating antioxidant activity from guava fruit extracts. Journal of Food Composition and Analysis 19: 669-675.

Thymi, S., Krokida, M.K., Papa, A. and Maroulis, Z.B., 2005. Structural properties of extruded corn starch. Journal of Food Engineering 68: 519-526.
Tudorică, C.M., Kuri, V. and Brennan, C.S., 2002. Nutritional and physicochemical characteristics of dietary fiber enriched pasta. Journal of Agricultural and Food Chemistry 50(2): 347-356.

Wandee, Y., Uttapap, D., Puncha-Arnon, S., Puttanlek, C., Rungsardthong, V. and Wetprasit, N., 2014. Enrichment of rice noodles with fibre-rich fractions derived from cassava pulp and pomelo peel. International Journal of Food Science and Technology 49: 2348-2355.

Yağcl, S. and Gögüus, F., 2008. Response surface methodology for evaluation of physical and functional properties of extruded snack foods developed from food-by-products. Journal of Food Engineering 86: 122-132. 
